\title{
Source Detection and Functional Connectivity of the Sensorimotor Cortex during Actual and Imaginary Limb Movement: A Preliminary Study on the Implementation of eConnectome in Motor Imagery Protocols
}

\begin{abstract}
Alkinoos Athanasiou, Chrysa Lithari, Konstantina Kalogianni, Manousos A. Klados, and Panagiotis D. Bamidis

Lab of Medical Informatics, Medical School, Aristotle University of Thessaloniki (AUTH), 54124 Thessaloniki, Greece

Correspondence should be addressed to Alkinoos Athanasiou, alkinoosathanassiou@gmail.com

Received 6 July 2012; Revised 29 November 2012; Accepted 29 November 2012

Academic Editor: Christos Papadelis

Copyright ( 2012 Alkinoos Athanasiou et al. This is an open access article distributed under the Creative Commons Attribution License, which permits unrestricted use, distribution, and reproduction in any medium, provided the original work is properly cited.

Introduction. Sensorimotor cortex is activated similarly during motor execution and motor imagery. The study of functional connectivity networks (FCNs) aims at successfully modeling the dynamics of information flow between cortical areas. Materials and Methods. Seven healthy subjects performed 4 motor tasks (real foot, imaginary foot, real hand, and imaginary hand movements), while electroencephalography was recorded over the sensorimotor cortex. Event-Related Desynchronization/Synchronization (ERD/ERS) of the mu-rhythm was used to evaluate MI performance. Source detection and FCNs were studied with eConnectome. Results and Discussion. Four subjects produced similar ERD/ERS patterns between motor execution and imagery during both hand and foot tasks, 2 subjects only during hand tasks, and 1 subject only during foot tasks. All subjects showed the expected brain activation in well-performed MI tasks, facilitating cortical source estimation. Preliminary functional connectivity analysis shows formation of networks on the sensorimotor cortex during motor imagery and execution. Conclusions. Cortex activation maps depict sensorimotor cortex activation, while similar functional connectivity networks are formed in the sensorimotor cortex both during actual and imaginary movements. eConnectome is demonstrated as an effective tool for the study of cortex activation and FCN. The implementation of FCN in motor imagery could induce promising advancements in Brain Computer Interfaces.
\end{abstract}

\section{Introduction}

Motor imagery (MI) is described as the concept of imagining a motor task without resulting in physical execution. It is the visualization and rehearsal of an imaginary movement [1] as opposed to the actual practice of the movement, which is described by the term Motor Execution (ME). This concept is widely applied in the development of Brain Computer Interfaces (BCIs). BCIs are systems that translate human volition to control of external devices and exploit a person's will to move or to communicate, regardless if that person is able to actually perform such a task due to severe impairments (such as spinal cord injury (SCI), stroke, or amyotrophic lateral sclerosis (ALS)) [2]. Cortical activation during motor imagery can be recorded with a variety of methods, including functional Magnetic Resonance Imaging (fMRI), which has the optimal spatial accuracy [3], and electroencephalography (EEG), which provides excellent temporal accuracy (on the order of milliseconds) [2]. EEG in particular has been extensively applied, as it is an inexpensive, widely available, and relatively simple method that can be applied to real-life scenarios [2].

The brainwaves can be extracted by the EEG easily and in real time and play a crucial role in BCI applications. The mu-rhythm has been identified as an EEG feature that corresponds to movement volition and is usually examined in motor imagery studies [4]. Its physiological role is not yet clearly defined, though it is associated with 
the inhibition of the movement [5]. The amplitude of the mu-rhythm typically decreases when the corresponding motor areas are activated and is most accurately recorded over the primary sensorimotor cortex [5]. Its exact range is not firmly defined, but it generally overlaps with the alpha rhythm $(8-12 \mathrm{~Hz})$ [6] and possibly with a part of the lower beta band [4]. Mu-rhythm (also known as sensorimotor rhythm (SMR)) is commonly studied using Event-Related Desynchronization/Synchronization analysis (ERD/ERS), where ERD usually denotes the activation of cortical areas, while ERS denotes a decrease in excitability and information processing [7].

BCIs continue to develop over the past five years becoming more user friendly, accurate, and efficient [8], while they still carry certain drawbacks that need to be addressed [9]. However, ERD/ERS of the EEG mu-rhythm provides spatially static neuroelectric information of brain regions that are activated during tasks. ERD/ERS analysis does not convey the information of how these regions communicate with each other [10]. Brain activity is distributed spatiotemporally, and brain functional networks are formed through this distribution. The behavior of such networks provides important physiological information for understanding brain functions and dysfunctions [11]. The relatively new concept of brain functional connectivity [11] promises to play a key role in neurosciences, allowing researchers to study the organized behavior of brain regions beyond the standard cortical source estimation, mapping, and localization of activity [12]. The estimation of functional cortical connectivity aims at describing the interactions between differently organized and specialized cortical regions as patterns depicting dynamics of information flow between those regions [10].

A conceptual definition of functional connectivity is stated as a "temporal correlation between spatially remote neurophysiological events" [10,13]. Several approaches have been proposed for the estimation of this correlation, known as connectivity metrics. Latest approaches, such as the Directed Transfer Function (DTF) and the Adaptive Directed Transfer Function (ADTF), rely on the key concept of Granger causality between time series [14]. The mathematical background of these methods lies beyond the scope of this paper, but —on a very short account - the lack of reciprocity between two times series, one of which results to the other, provides the direction of the information flow between each pair of elements [15].

In our work, a classic paradigm of cue-paced motor imagery is deployed in healthy young volunteers. Our goal is to investigate the implementation of functional connectivity on Brain Computer Interfaces and Motor Imagery protocols and to examine the possibility of classifying the motor volition accurately and fast by functional connectivity analysis. For this purpose, we used the novel MATLAB toolbox, eConnectome [16]. The solutions to the inverse problem were provided by the toolbox together with the activation maps on cortical level. We currently present the preliminary results of implementing functional connectivity analysis on motor imagery by exploiting the open eConnectome toolbox.

\section{Materials and Methods}

In the current study 7 healthy right-handed subjects participated (4 male and 3 female); mean age of 28.1 (range 23-37). The procedure was accurately explained beforehand, and all subjects gave their written consent prior to the experimental procedure. None of the subjects had any experience in the concept of motor imagery prior to their participation. The setup of the experiment and part of the analysis has been analytically described in our previous work [17]. Each subject performed four motor tasks: (a) real hand movement (biceps-flexion of the forearm), (b) imaginary hand movement, (c) real foot movement (quadricepsstretch of the lower leg), and (d) imaginary foot movement. Each task was repeated 95 times, divided into five sets of 19 trials each. There was 1-minute rest between sets and 5minute rest between tasks. During trials, the subjects were presented with visual feedback on a computer screen (the word "move"), which constituted the cue to perform the relevant task. The cue of the visual feedback was recorded with an optic fiber placed on the computer screen serving as the trigger channel synchronous to the EEG recording. A Nihon-Kohden (Japan) EEG and an active electrode cap (EASYCAP, Germany) were used. EEG was recorded by 17 electrodes $(\mathrm{CP} 3, \mathrm{CP} 1, \mathrm{CPz}, \mathrm{CP} 2, \mathrm{Cp} 4, \mathrm{C} 5, \mathrm{C} 3, \mathrm{C} 1, \mathrm{Cz}$, $\mathrm{C} 2, \mathrm{C} 4, \mathrm{C} 6, \mathrm{FC} 3, \mathrm{FC} 1, \mathrm{FCz}, \mathrm{FC} 2$, and $\mathrm{FC} 4$ ), placed in accordance with the 10-10 international electrode system. The recording electrodes were referenced with LPA and RPA mastoid electrodes. The impedance threshold was set below $5 \mathrm{kOhm}$. The electrode setup corresponds to the skull area above the sensorimotor cortex.

Following signal extraction, further processing is described in two parts. Initially, the extracted signals were processed with an ERD/ERS of mu-rhythm EEG paradigm in mind. This paradigm of cue-paced motor imagery [17], is presented here as an intermediate step that serves to instigate our research towards functional connectivity. The second part, consisting of source depiction and functional connectivity of the extracted signals, deploys different processing methodology, as we describe accordingly. Signal preprocessing and analysis was performed in MATLAB (Mathworks Inc.), using EEGLAB toolbox [18] and eConnectome toolbox [16].

For ERD/ERS analysis, we chose to focus on 7 electrodes (CP1, CPz, CP2, C1, Cz, C2, FC1, FCz, and FC2), those more relevant to the primary motor cortical areas of hand and foot. Filtering was performed using EEGLAB [16] at 8-15 Hz (mu-rhythm, possibly including a fraction of the lower beta band) [4]. Independent Component Analysis (ICA) was used to remove ocular artifacts. Epochs were set from $800 \mathrm{msec}$ prestimulus to $2200 \mathrm{msec}$ poststimulus.

Following preprocessing, Event-Related Desynchro-nization/Synchronization (ERD/ERS) values of the mu-rhythm were calculated. Each subject's 95 epochs for each task were first divided in five sets of 19 epochs and then were averaged across sets. For each of the five sets, as well as for each of the seven focus electrodes, ERD/ERS was computed at three poststimuli intervals (100-400 msec, $400-700 \mathrm{sec}$, and 700$1000 \mathrm{msec}$ ) towards $-300-0 \mathrm{msec}$ prestimulus, to account 
for different reflexes of the subjects. This resulted in matrices of $7 \times 5 \times 3$ size, containing the ERD/ERS values for each subject. In order to test for similarities between the ERD/ERS values during the real movements and ERD/ERS values during the imaginary ones, Student's $t$-tests were performed. Moreover, the ERD/ERS values during the imaginary foot movements were compared to their counterparts during the imaginary hand movements to reveal any differentiation. For all statistical tests, the level of significance was set to 0.05 .

Source imaging and functional connectivity on the source level were applied taking into account all 17 electrodes. The area covered by the electrodes can be anatomically corresponded to the primary motor area, primary somatosensory area, and premotor cortex of the brain. In order to append those signals recorded at the surface of the skull to specific Brodmann areas of the cortex though, the solutions to the "inverse problem" have to be calculated. eConnectome handles this part using the Cortical Current Density (CCD) source model [19]. The toolbox provides a high-resolution cortical surface model segmented and reconstructed for visualization from MRI images of the Montreal Neurological Institute and a scalp surface that forms the sensor space. These are generic realistic head models designed to provide improved accuracy in cortical source estimation [20]. Cortical regions of interest (ROIs), corresponding to Brodmann areas, are predefined (by the eConnectome software) and available to compute estimated cortical sources. The user has also the choice to define custom regions of interest, a feature that we explored in our study.

Preprocessing involved filtering at $8-15 \mathrm{hz}$, and ICA using EEGLAB [16] and epochs were set from $800 \mathrm{msec}$ prestimulus to $2200 \mathrm{msec}$ poststimulus. At this point, one further step was decided, in order to compute the average of all (95) epochs for each subject-rather than in sets of 19 (as was done for the ERD/ERS analysis). This produced a dataset of four EEG sets for each of the seven subjects. Each of these four sets consisted of the average of 95 trials during each task: Foot Motor Execution (FME), Foot Motor Imagery (FMI), Hand Motor Execution (HME), and Hand Motor Imagery (HMI). Using the EEG module of eConnectome [21], for each subject, we compared ROI activation of FME versus FMI, HME versus HMI, as well as FME versus HME, and FMI versus HMI. The average of each task trials for each subject was chosen over individual epoch analysis, in order to minimize the effect of random occurrences and artifacts to the imaging of cortical activation. Similarly, it is suggested that the causality relations between cortical networks appear to be independent from the frequency band analyzed [10]. Thus, we opted to estimate source activation for the narrow frequency band that we used for mu-rhythm ERD/ERS analysis and connectivity was computed for the band of murhythm (8-12 hz), aiming for better comparability of results and reduction of computational workload.

Directed Transfer Function (DTF) was used for the estimation of functional connectivity relationships between cortical areas of the human brain [16]. DTF is a connectivity metric based on the multivariate autoregressive (MVAR) modeling [22]. It was applied on the whole -800 to $2200 \mathrm{msec}$ interval for the range of $8-12 \mathrm{~Hz}(\mathrm{mu}$ or
TABLE 1: Real/imaginary statistical similarity and hand/foot discrimination percentages of subjects using $t$-test comparisons $(P>$ $0.05)$ of ERD/ERS values: our subjects showed high performance in Foot and Hand Motor Imagery but low source discrimination.

\begin{tabular}{ll}
\hline Real/imaginary similarity & \\
Hand & $85.71 \%$ \\
Foot & $71.41 \%$ \\
Hand/foot discrimination & \\
Real & $28.57 \%$ \\
Imaginary & $14.28 \%$ \\
\hline
\end{tabular}

alpha rhythm). For a single representative subject, the functional connectivity of the sensorimotor cortex during motor execution and imagery for both foot and hand movements was analyzed and further compared. During this investigation, we custom defined ROIs on the sensorimotor cortex, correlating to primary hand and foot motor areas (M1), hand and foot sensory areas (S1), and supplementary motor areas (SMAs). The selected subject was the one with the highest performance in both FMI and HMI, as defined by no differentiation between imaginary and real ERD/ERS patterns in the majority of focus electrodes and time intervals. Connectivity patterns were visualized at both the cortical surface and the EEG sensor level, on a model using the standard 10-10 electrode system [23].

\section{Results}

3.1. Event-Related Desynchronization/Synchronization (ERD/ ERS) Analysis. Regarding the ability to perform motor imagery, four out of seven subjects performed equally well in both FMI and HMI, activating their cortex during imaginary movements in the same patterns as in real movements $(P>$ 0.05 ) in all of the three time intervals as reported previously by our group [17].

Regarding the ability to statistically discriminate between hand and foot movements, in two out of seven subjects, ERD/ERS of the mu-rhythm was unable to provide handfoot discrimination in either MI or ME. For two subjects, hand-foot discrimination (for both MI and ME) was poor, both spatially (7 electrodes) and temporally (3 time intervals). For further two subjects, the discrimination between foot and hand ME was possible across most electrodes and time intervals. Finally, one subject produced distinctive ERD/ERS patterns for foot and hand MI, while this was not the case for ME.

The results of ERD/ERS statistical analysis, published in a previous paper [17] and presented here in a summary (Table 1), encouraged us to further investigate MI movements in terms of functional connectivity.

3.2. Cortical Source Imaging. The distinction between different cortical sources' activation is facilitated with the visualization of the inverse problem solutions (computed with Current Cortical Density) that provide the corresponding cortical activation maps. In all subjects, as expected, visual discrimination between foot and hand activation 

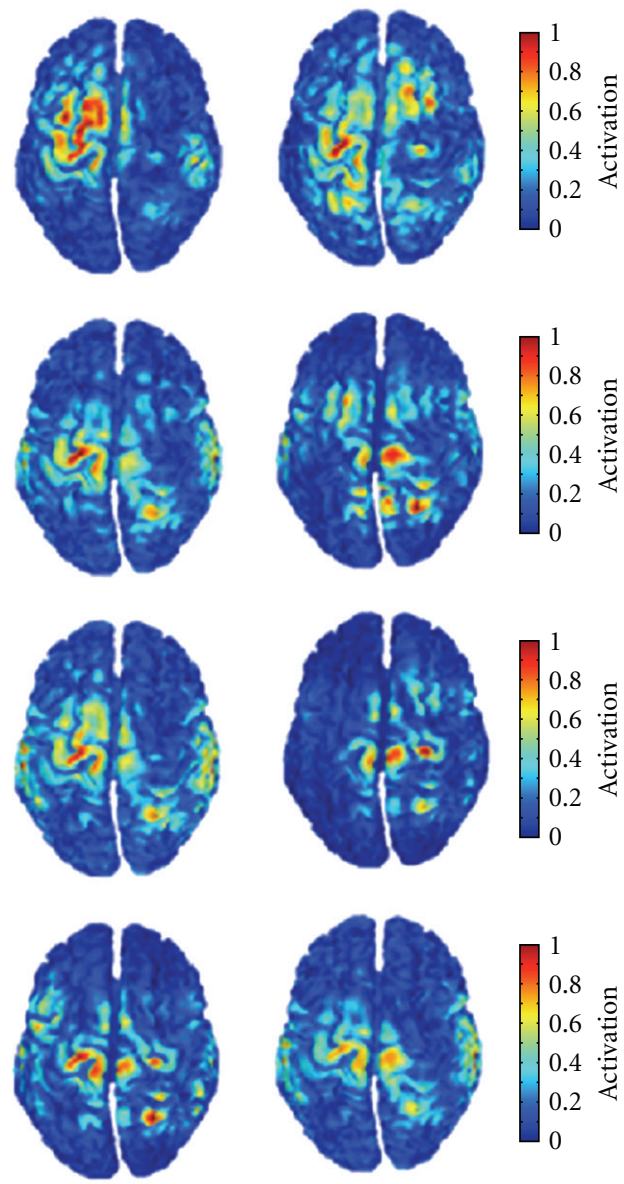

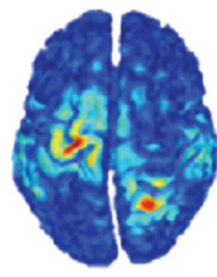

(a) HMI

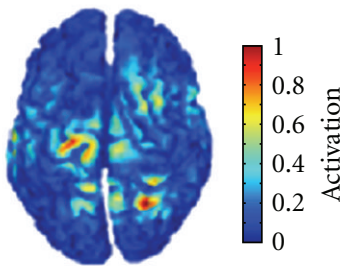

(b) FMI
Figure 1: Cortical source activation maps produced by eConnectome after solving the inverse problem using Current Cortical Density. Poststimuli instances for 5 subjects during (a) Hand Motor Imagery (HMI) and (b) Foot Motor Imagery (FMI). Different activation patterns can be observed for different subjects, but in all of them, the hand and foot imagery are discriminated.

in real movements is apparent. Moreover, in five out of seven subjects, the discrimination becomes possible between imaginary foot and hand movements as well (Figure 1). As also shown in Figure 1, not all subjects activate the relevant cortical sources in the same pattern, especially during imaginary movement. It can be observed that during imaginary movements, there is greater involvement of the ipsilateral hemisphere. Moreover, activation patterns are not static during the whole epoch (the average of 95 epochs of each task), but different instances produce different patterns for each subject in both MI and ME tasks (Figure 2).
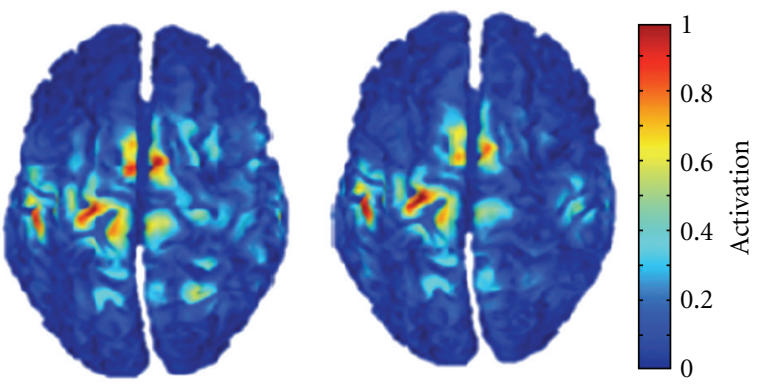

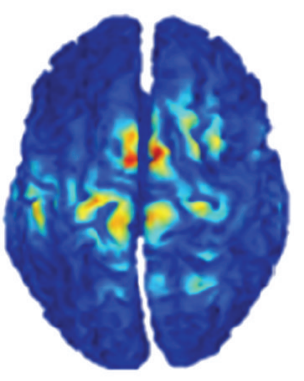

(a) HMI

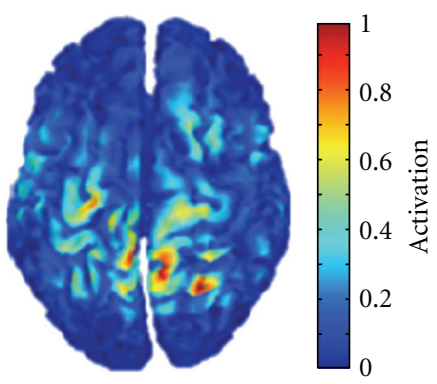

(b) HME
FIGURE 2: Cortical source activation maps for one subject during (a) Hand Motor Imagery (HMI) and (b) Hand Motor Execution (HME). Each row represents a different poststimulus instance, and the variance in activation patterns can be observed.

Although activation is similar during MI and ME, generally imagery produces lower levels of activation, at both scalp and cortical area (Figure 3). Finally, it can be observed that activation of supplementary motor areas (SMAs) varies and is more intense during foot motor for all subjects. This is demonstrated at Figure 4 for subject 1, but similar patterns stand for the rest of the subjects.

3.3. Functional Connectivity. For this part of our analysis, one representative subject was chosen, as described in Section 2. All figures hereafter concern this particular subject. Functional connectivity can be studied either at the scalp surface (electrode plane) or at the cortical surface (regions of interest plane). Figure 5 depicts connectivity at the electrode plane, at different instances for one subject. Different connectivity patterns can be recognized at different temporal instances, but there are some that prevail across the whole task as shown in the second and the third rows of Figure 5. In HME, a strong outflow current is produced from electrode $\mathrm{C} 1$ towards electrodes corresponding to both hemispheres of the sensorimotor cortex (Figure 5(a)). eConnectome provides the option to portray information flow between all channels or the single highest outflow, inflow, or information exchange. This outflow is maximal towards CP4 and FC2 in most examined instances. On the contrary, in HMI, this strong outflow is not as obvious, although when each electrode is examined, the same prevalence of $\mathrm{C} 1$ can be found. In HMI, information flow is portrayed to be maximal from electrode $\mathrm{C} 1$ towards electrode $\mathrm{FC} 2$ and from electrode FC1 towards electrode C4 (Figure 5(b)). 


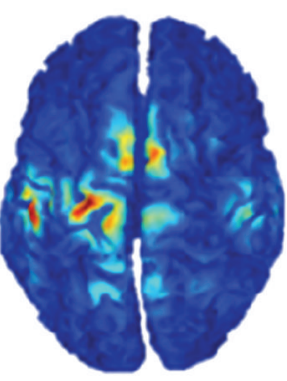

(a) HMI

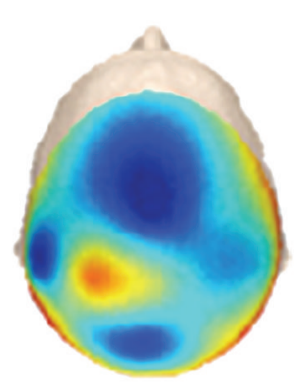

(c) HMI

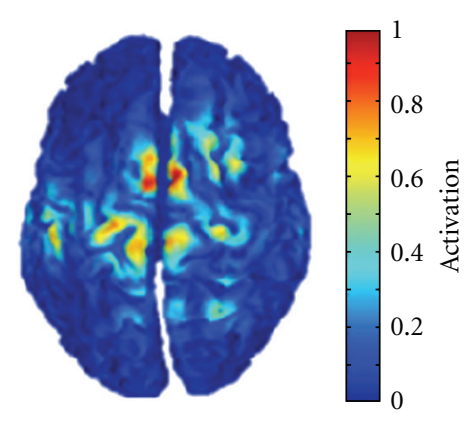

(b) HME

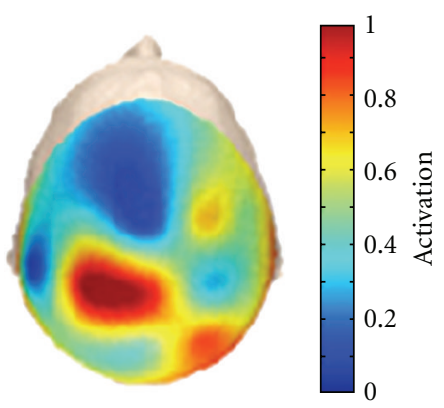

(d) HME
FIGURE 3: First row: cortical source activation maps for (a) Hand Motor Imagery (HMI) and (b) Hand Motor Execution (HME). Second row: scalp area activation maps for (c) HMI and (d) HME. All images are from a single subject, and a lower level of activation can be observed in imagery as compared to the corresponding instance during execution.

On the cortical surface, after solving the inverse problem, functional connectivity can be studied either using the predefined regions of interest (cortical ROIs defined by eConnectome, corresponding to Brodmann areas) or using custom-defined ROIs on the dynamic activation maps produced by source imaging. Initially, the primary hand motor areas were defined in both hemispheres, and then Directed Transfer Function (DTF) was computed. Figure 6 depicts a strong information flow between primary hand motor areas, directed from the contralateral (to the moving hand) towards the ipsilateral area. This flow is almost identical in power amplitude in both motor execution and motor imagery. To test whether that was a result of defining only primary motor areas, another ROI corresponding to primary right foot sensory cortex (the purple-colored area in Figure 6(b)) was defined, but no information flow was detected. In Figure 7, the cortical network produced by Hand Motor Imagery consists, additionally, of the SMAs and the primary hand sensory areas. In this case, information flow is stronger from the SMAs towards the ipsilateral primary hand motor area, showing the important regulative role of those cortical sources in the imagination (preparation) of a movement [10]. The activation time series of the ROIs of this small cortical network, consisting of primary hand motor and sensory areas and SMAs, is presented in Figure 8. Finally, another small cortical network was tested,

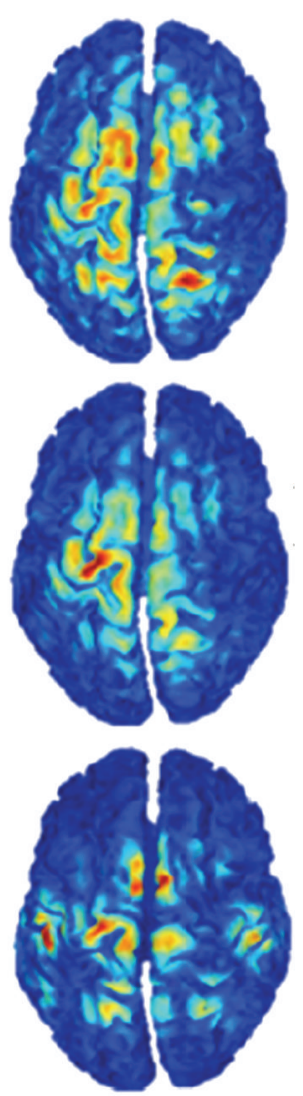

(a) FMI
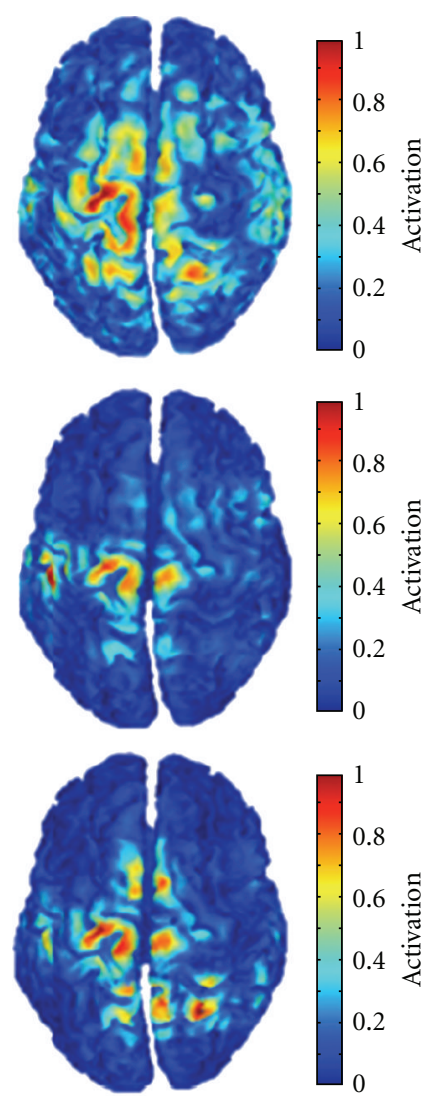

(b) FME
FIgure 4: Cortical source activation maps for one representative subject during (a) Foot Motor Imagery (FMI) and (b) Foot Motor Execution (FME). The activation of supplementary motor areas (SMAs) varies in different poststimulus instances (rows) and can be observed to be more intense during FMI.

this time consisting of primary foot motor areas and SMAs. The information flow from the SMAs is present in this network too, directed to the contralateral primary foot motor area. In addition, there is a high output information exchange between the SMAs of both hemispheres as shown in Figure 9.

\section{Discussion}

The sensorimotor cortex, during the practice of motor imagery tasks, has been proven to produce similar patterns of activation with actual motor execution tasks $[4,6]$, something our work also confirms [17]. Cortex activation is found to be less intense during motor imagery as compared to motor execution, which is shown by ERD/ERS amplitude as well as by source activation maps produced by eConnectome. Mu-rhythm detection and ERD/ERS analysis achieve high performance (over $85 \%$ for Hand Motor Imagery in our experiment) and are considered to be a suitable modality for accurate and fast classification of human motor volition. However, ERD/ERS analysis does not facilitate the discrimination between different sources' 

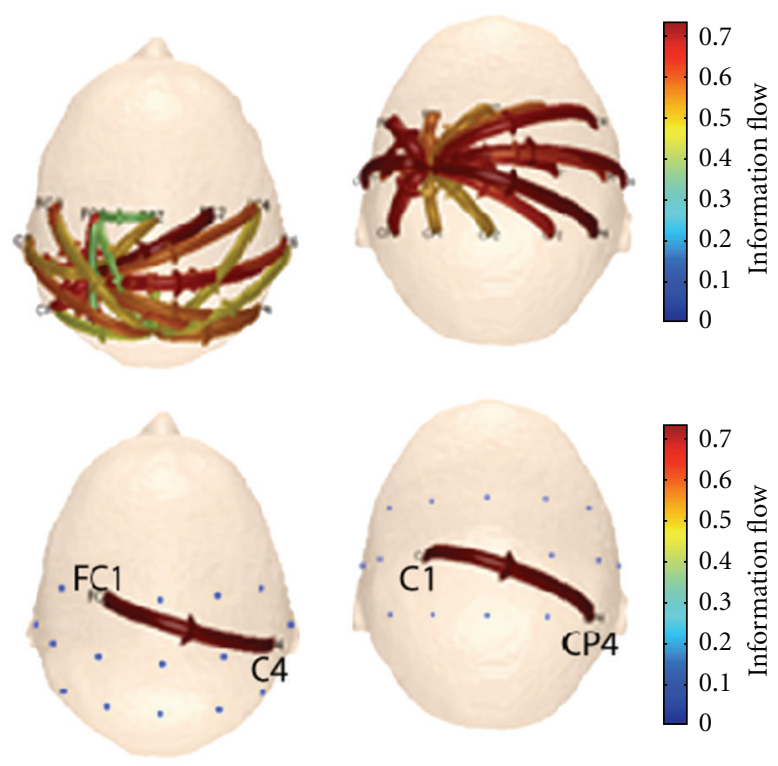

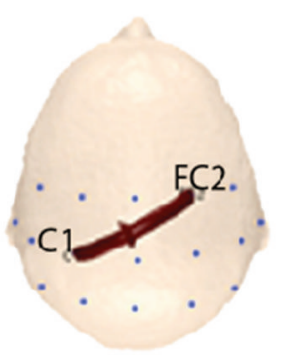

(a) HMI

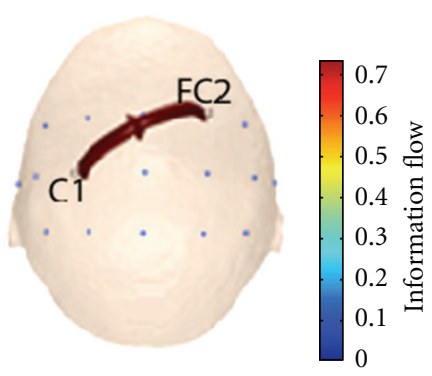

(b) HME
FIGURE 5: Functional connectivity patterns at the electrode plane for one representative subject during (a) Hand Motor Imagery (HMI) and (b) Hand Motor Execution (HME). First row: information exchange between all channels. Second and third rows: maximal information exchange.

activation. On the contrary, the production of cortical source activation maps, such as those produced by eConnectome, can be greatly effective in source discrimination. As has been proposed by BCI experts, design of motor restoration systems should be based on a self-paced (asynchronous) approach [24]. Our results agree with this viewpoint, as each subject tended to produce unique patterns of brain activity, regarding electrodes and time intervals of activation [17]. The study of functional connectivity at both the scalp and the source level can help further understanding of each subject's unique patterns, providing information on "communication" exchange between electrodes and cortical sources.

The mu-specific approach was induced by certain reasons. Concerning sensorimotor-related rhythms, it is proposed that mu- and beta-rhythms are couple-phased and should not be combined as independent control features [25]. Also, it is suggested that causality relation between cortical networks is not maximally present in any specific frequency band [10]. Taking those viewpoints into account allowed us to focus on a narrow band of brain activity, reducing computational workload, while at the same time without resulting in sacrifice of information. Our brain connectivity findings tend to confirm novel studies in the area [10, $15,21]$ regarding the highly dynamic nature of functional connectivity networks [15]. Such studies report small-world topologies [11] in brain networks, a characteristic that seems to play a crucial role regarding neurophysiological organization and behavior of the cortex [26, 27] during motor execution and imagery. Furthermore, in the current study, eConnectome revealed the SMAs as a clearly defined nested network exchanging information with primary motor regions, a finding we appreciate to be coherent with the regulative role of SMAs during the planning of a movement [10]. Through the use of eConnectome, we were able to study specific cortical regions of interest and the way they interact in motor execution and motor imagery tasks of the hand and the foot.

There are several limitations that have to be mentioned, including some that are not specific to our study. Primarily, the low number of subjects along with the absence of patients does not allow us to safely generalize our findings and conclude an effective BCI approach for mobility restoration. Moreover, regarding connectivity analysis, we have to mention again that in the view of the feasibility analysis aimed at in this paper, only exploratory results are reported herein, based on the investigation of ME and MI performance for one subject. However, we reiterate that this study dealt with the feasibility and usefulness of BCI and connectivity integration rather than with specific neurophysiological findings. There are many changes in brain activation and functional connectivity that come with severe impairment $[8,10]$. As such, the inclusion of patients in such research is crucial in order to produce useful conclusions.

Regarding our method, the absence of electromyographic (EMG) signal recording is crucial to further analysis, since the trials were not accurately time locked, and the contamination because of muscular artifacts could not be eliminated. Also, a higher number of EEG sensors would have resulted to better spatial resolution, and in order to efficiently study connectivity, we ought to have a recording of higher spatial resolution (more electrodes, possibly placed in accordance with 10-5 international system [23]). However, an increased number of EEG sensors have been identified to produce unwanted (false) small-world topologies [11], since uncorrelated electrodes would be more easily influenced by strong focal signals. Practicality reasons also demand that a possible BCI-FCN integration should deploy a rather small number of electrodes in order to facilitate portability and usage in real-life scenarios. The optimal compromise between portability and spatial resolution is something that has yet to be researched.

Finally, the selection of the connectivity metric is proven to be critical for the formation of the brain functional networks [28], and a more reliable interpretation of measured activations and information flow could be obtained by comparing results from different connectivity metrics. Furthermore, eConnectome includes specific multivariate metrics (DTF and ADTF) that are not proven to be 


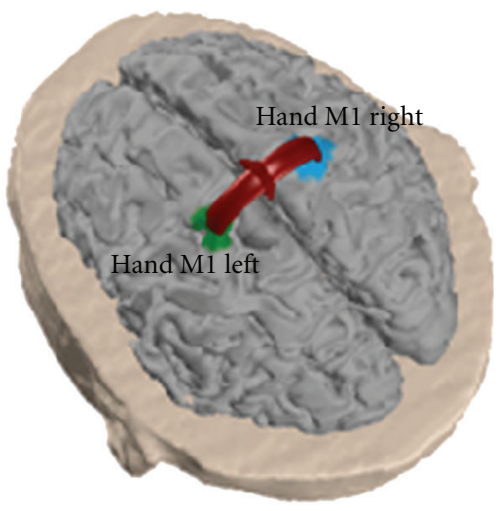

(a) HME

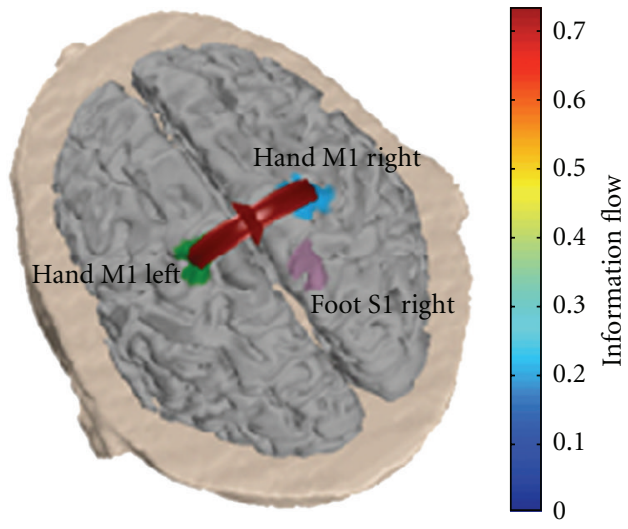

(b) HMI

FIGURE 6: Identical high Information outflow from the cortical ROI corresponding to hand area of motor cortex contralateral to movement towards the same ipsilateral area during (a) Hand Motor Execution (HME) and (b) Hand Motor Imagery (HMI).

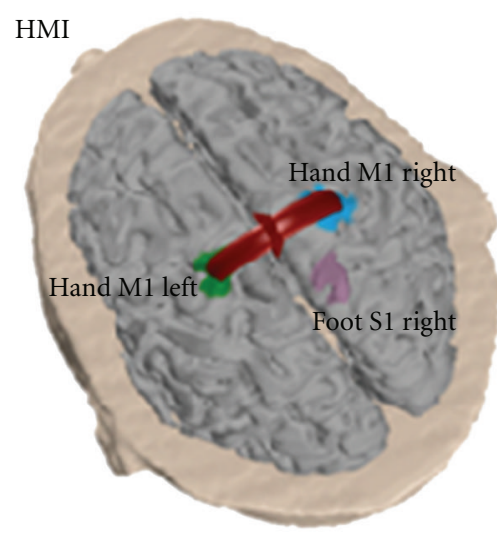

(a)

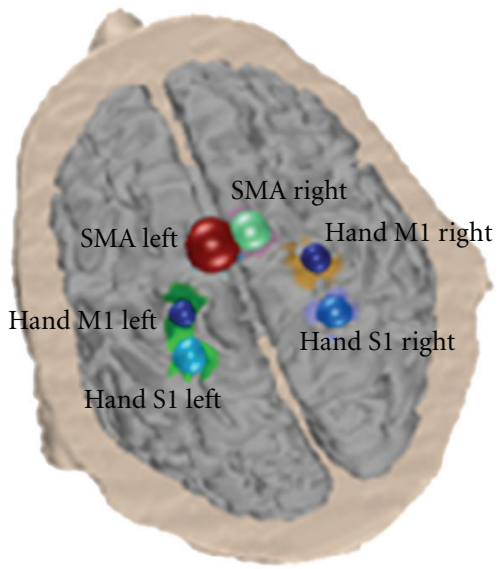

(c)

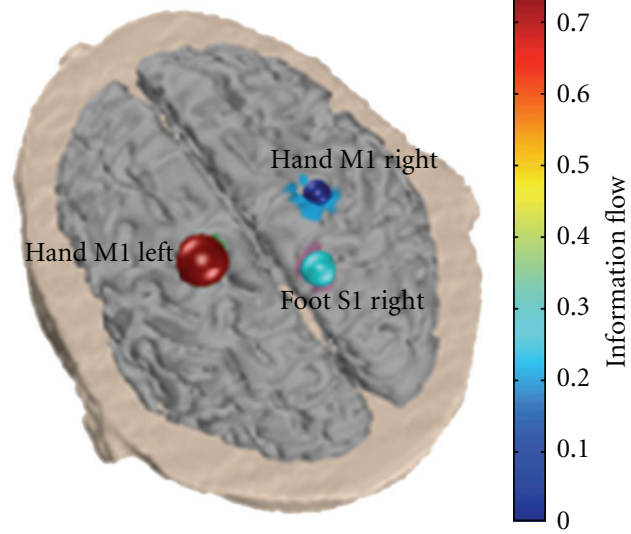

(b)

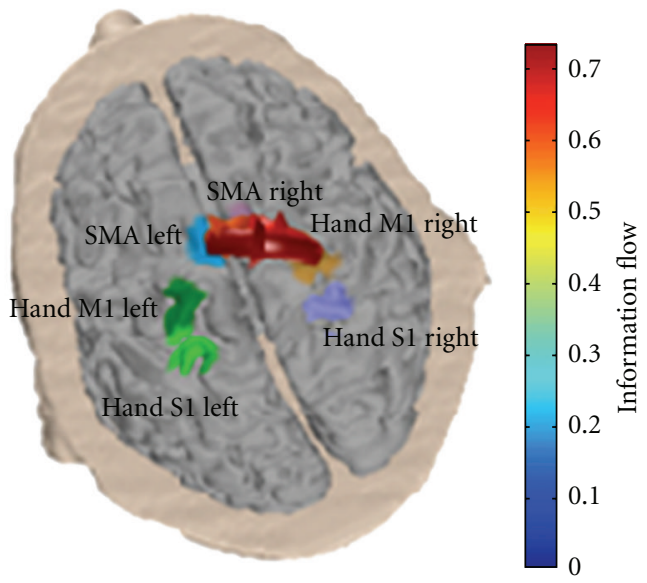

(d)

FIGURE 7: FCN during Hand Motor Imagery (HMI) of one representative subject. (a) Information exchange between primary hand motor areas, (b) information outflow from the same areas, (c) information outflow from SMAs, and (d) the highest information exchange was shown to occur between the two SMAs and the ipsilateral primary hand motor area. The size of the spheres is proportional to the outflow, which is also obvious from the chromatic scale. 

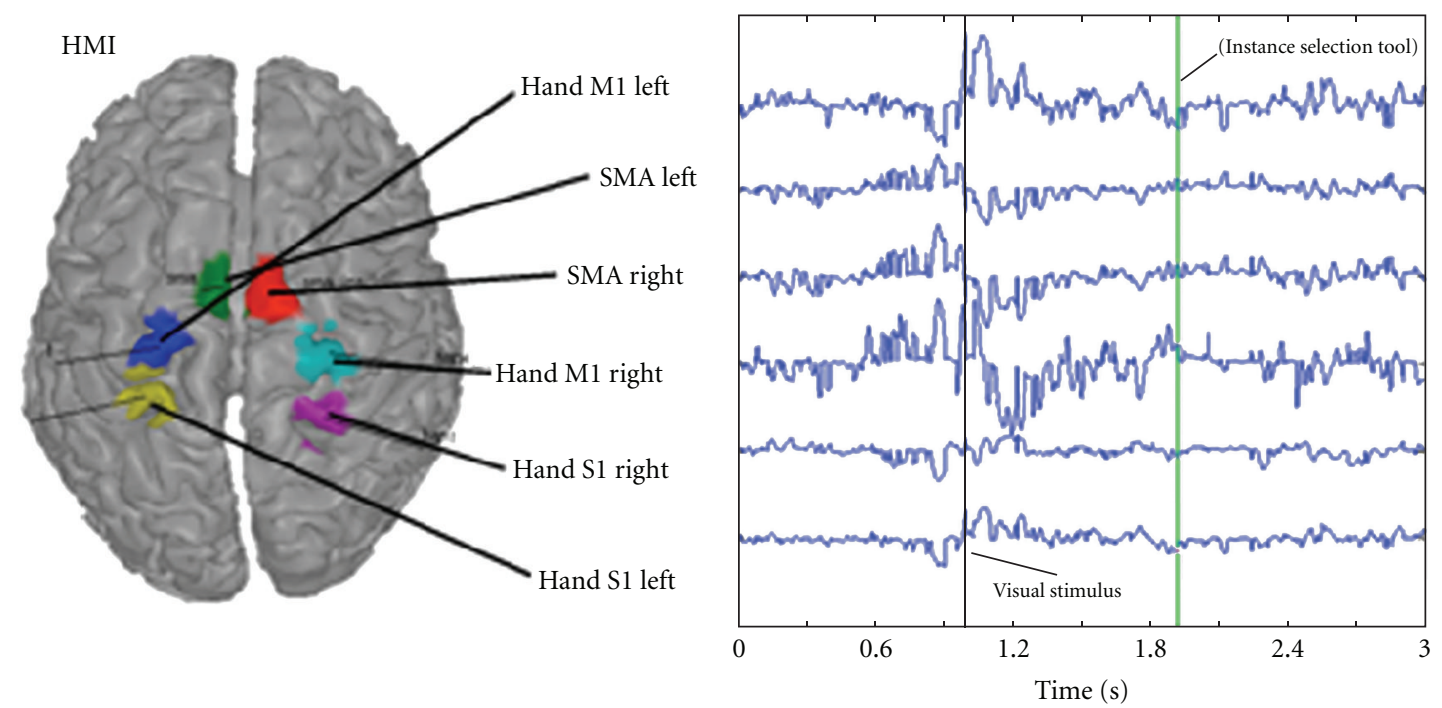

FIGURE 8: Regions of interest activation time series of a small functional cortical network (FCN) of one representative subject during Hand Motor Imagery (HMI).
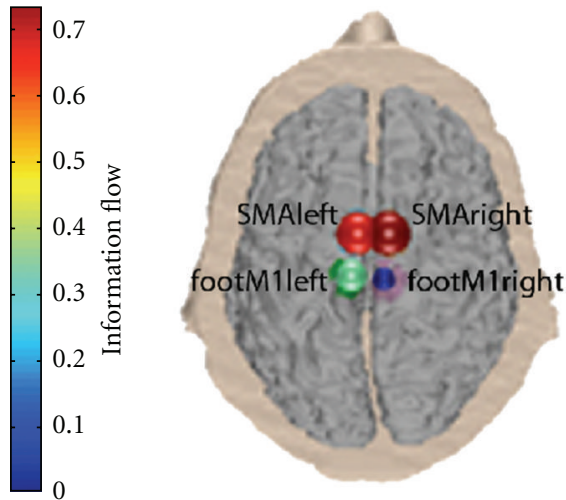

(a) FME

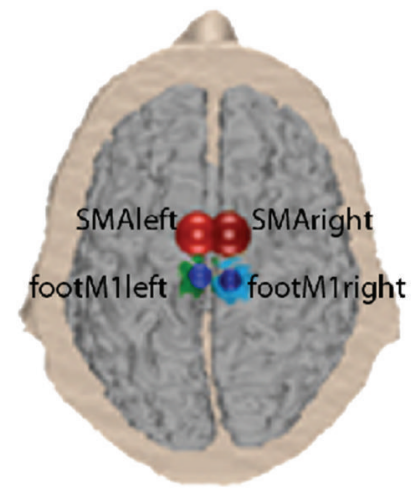

(b) FMI

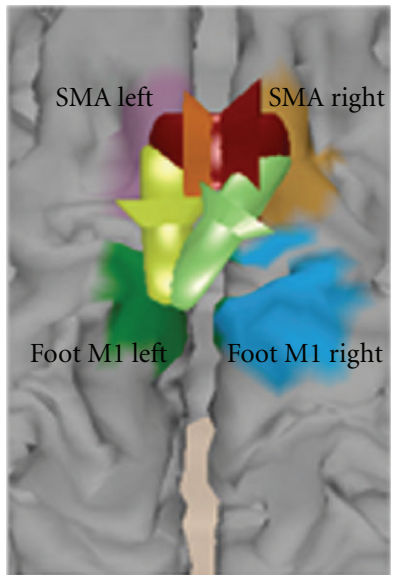

(c) FMI

FIGURE 9: FCNs of one representative subject during foot motor tasks. Information outflow from SMAs and primary foot motor areas during (a) Foot Motor Execution (FME) and (b) Foot Motor Imagery (FMI). (c) Information exchange of those areas during Foot Motor Imagery (zoom in).

specifically more suitable for BCI approaches than other connectivity metrics. The integration of connectivity in Brain Computer Interfaces seems far from being immediately feasible. It has to be stressed that DTF cannot yet be performed in real time. The prospect of real-time application of a connectivity metric in a BCI application would also require a time-adaptive metric, such as the ADTF, which seems more suitable for such a use. However, ADTF needs even more computational workload and time to be performed than the DTF. The real-time environment that is crucial for rehabilitation or commercial BCI approaches cannot yet benefit from connectivity analysis, though this is a limitation that time and technological progress is expected to eventually solve [29].
In conclusion, it is our conviction that the study of functional connectivity in the context of Brain Computer Interfaces applications, although not yet a mature solution, has a lot to offer in terms of neurophysiological integration and understanding and the design of intuitive high-performance self-paced systems. eConnectome is an appropriate toolbox to be used in order to test and enhance the effectiveness of Motor Imagery protocols and Brain Computer Interface systems in a research environment. In our study, this toolbox enabled us to extensively study and detect expected similarities and differences in cortical activation, as well as depict simple FCNs between a small number of highly important cortical regions of interest. eConnectome is demonstrated to be able to facilitate the study of functional connectivity, 
providing easy-to-use tools and graphical interpretations of cortical activity and information exchanges. Further evaluation and experiments involving healthy and patient subjects need to be performed in order to fully implement FCN findings in typical BCIs. We cannot emphasize enough the breakthroughs that can occur in the field of motor restoration; however, we can only capture the need towards the design of appropriate software and tools to precipitate the integration of the two fields of research.

\section{References}

[1] J. Decety and D. H. Ingvar, "Brain structures participating in mental simulation of motor behavior: a neuropsychological interpretation," Acta Psychologica, vol. 73, no. 1, pp. 13-34, 1990.

[2] A. Athanasiou and P. D. Bamidis, "A review on brain computer interfaces: contemporary achievements and future goals towards movement restoration," Aristotle University Medical Journal, vol. 37, no. 3, pp. 35-44, 2010.

[3] R. Sitaram, A. Caria, R. Veit et al., "FMRI brain-computer interface: a tool for neuroscientific research and treatment," Computational Intelligence and Neuroscience, vol. 2007, Article ID 25487, 10 pages, 2007.

[4] G. Pfurtscheller and C. Neuper, "Motor imagery activates primary sensorimotor area in humans," Neuroscience Letters, vol. 239, no. 2-3, pp. 65-68, 1997.

[5] S. Arroyo, R. P. Lesser, B. Gordon, S. Uematsu, D. Jackson, and R. Webber, "Functional significance of the mu rhythm of human cortex: an electrophysiologic study with subdural electrodes," Electroencephalography and Clinical Neurophysiology, vol. 87, no. 3, pp. 76-87, 1993.

[6] C. Neuper, R. Scherer, S. Wriessnegger, and G. Pfurtscheller, "Motor imagery and action observation: modulation of sensorimotor brain rhythms during mental control of a braincomputer interface," Clinical Neurophysiology, vol. 120, no. 2, pp. 239-247, 2009.

[7] E. V. C. Friedrich, D. J. McFarland, C. Neuper, T. M. Vaughan, P. Brunner, and J. R. Wolpaw, "A scanning protocol for a sensorimotor rhythm-based brain-computer interface," Biological Psychology, vol. 80, no. 2, pp. 169-175, 2009.

[8] J. Zhou, J. Yao, J. Deng, and J. P. A. Dewald, "EEG-based classification for elbow versus shoulder torque intentions involving stroke subjects," Computers in Biology and Medicine, vol. 39, no. 5, pp. 443-452, 2009.

[9] V. Morash, O. Bai, S. Furlani, P. Lin, and M. Hallett, "Classifying EEG signals preceding right hand, left hand, tongue, and right foot movements and motor imageries," Clinical Neurophysiology, vol. 119, no. 11, pp. 2570-2578, 2008.

[10] L. Astolfi, H. Bakardjian, F. Cincotti et al., "Estimate of causality between independent cortical spatial patterns during movement volition in spinal cord injured patients," Brain Topography, vol. 19, no. 3, pp. 107-123, 2007.

[11] A. A. Ioannides, "Dynamic functional connectivity," Current Opinion in Neurobiology, vol. 17, no. 2, pp. 161-170, 2007.

[12] F. Varela, J. P. Lachaux, E. Rodriguez, and J. Martinerie, "The brainweb: phase synchronization and large-scale integration," Nature Reviews Neuroscience, vol. 2, no. 4, pp. 229-239, 2001.

[13] K. J. Friston, "Functional and effective connectivity in neuroimaging: a synthesis," Human Brain Mapping, vol. 2, no. 1-2, pp. 56-78, 1994.
[14] C. W. J. Granger, "Investigating causal relations by econometric models and cross spectra methods," Econometrica, vol. 37, pp. 424-438, 1969.

[15] F. de Vico Fallani, L. Astolfi, F. Cincotti et al., "Cortical functional connectivity networks in normal and spinal cord injured patients: evaluation by graph analysis," Human Brain Mapping, vol. 28, no. 12, pp. 1334-1346, 2007.

[16] B. He, Y. Dai, L. Astolfi, F. Babiloni, H. Yuan, and L. Yang, "eConnectome: a MATLAB toolbox for mapping and imaging of brain functional connectivity," Journal of Neuroscience Methods, vol. 195, no. 2, pp. 261-269, 2011.

[17] A. Athanasiou, E. Chatzitheodorou, K. Kalogianni, C. Lithari, I. Moulos, and P. D. Bamidis, "Comparing sensorimotor cortex activation during actual and imaginary movement," in Proceedings of the 12th Mediterranean Conference on Medical and Biological Engineering and Computing (MEDICON '10), vol. 29 of IFMBE Proceedings, pp. 111-114, Chalkidiki, Greece, May 2010.

[18] A. Delorme and S. Makeig, "EEGLAB: an open source toolbox for analysis of single-trial EEG dynamics including independent component analysis," Journal of Neuroscience Methods, vol. 134, no. 1, pp. 9-21, 2004.

[19] A. M. Dale and M. I. Sereno, "Improved localization of cortical activity by combining EEG and MEG with MRI cortical surface reconstruction: a linear approach," Journal of Cognitive Neuroscience, vol. 5, no. 2, pp. 162-176, 1993.

[20] P. A. Valdés-Hernández, N. von Ellenrieder, A. OjedaGonzalez et al., "Approximate average head models for EEG source imaging," Journal of Neuroscience Methods, vol. 185, no. 1, pp. 125-132, 2009.

[21] F. Babiloni, F. Cincotti, C. Babiloni et al., "Estimation of the cortical functional connectivity with the multimodal integration of high-resolution EEG and fMRI data by directed transfer function," NeuroImage, vol. 24, no. 1, pp. 118-131, 2005.

[22] C. Wilke, L. Ding, and B. He, "Estimation of time-varying connectivity patterns through the use of an adaptive directed transfer function," IEEE Transactions on Biomedical Engineering, vol. 55, no. 11, pp. 2557-2564, 2008.

[23] R. Oostenveld and P. Praamstra, "The five percent electrode system for high-resolution EEG and ERP measurements," Clinical Neurophysiology, vol. 112, no. 4, pp. 713-719, 2001.

[24] R. Scherer, A. Schloegl, F. Lee, H. Bischof, J. Janša, and G. Pfurtscheller, "The self-paced graz brain-computer interface: methods and applications," Computational Intelligence and Neuroscience, vol. 2007, Article ID 79826, 9 pages, 2007.

[25] D. J. Krusienski, G. Schalk, D. J. McFarland, and J. R. Wolpaw, "A $\mu$-rhythm matched filter for continuous control of a brain-computer interface," IEEE Transactions in Biomedical Engineering, vol. 54, no. 2, pp. 273-280, 2007.

[26] F. Pichiorri, F. De Vico Fallani, F. Cincotti et al., "Sensorimotor rhythm-based brain-computer interface training: the impact on motor cortical responsiveness," Journal of Neural Engineering, vol. 8, no. 2, Article ID 025020, 2011.

[27] S. H. Jin, P. Lin, and M. Hallet, "Reorganization of brain functional small-world networks during finger movements," Human Brain Mapping, vol. 33, no. 4, pp. 861-872, 2012.

[28] F. Wendling, K. Ansari-Asl, F. Bartolomei, and L. Senhadji, "From EEG signals to brain connectivity: a model-based evaluation of interdependence measures," Journal of Neuroscience Methods, vol. 183, no. 1, pp. 9-18, 2009. 
[29] T. Li, J. Hong, and J. Zhang, "Electroencephalographic (EEG) control of cursor movement in three-dimensional scene based on small-world neural network," in Proceedings of the IEEE International Conference on Intelligent Computing and Intelligent Systems (ICIS '10), vol. 3, pp. 587-591, Xiamen, China, October 2010. 

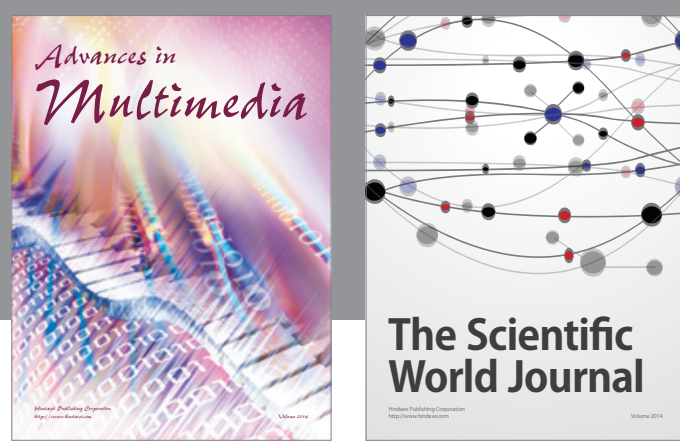

The Scientific World Journal
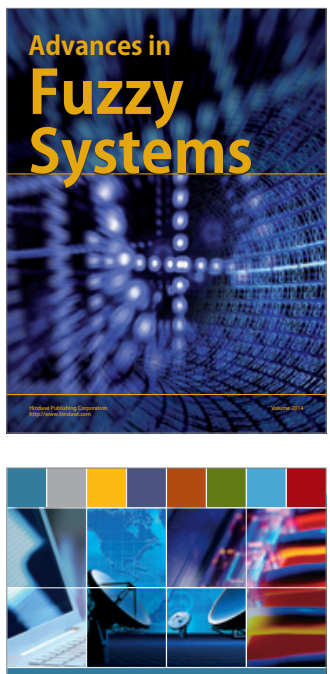

Computer Networks and Communications
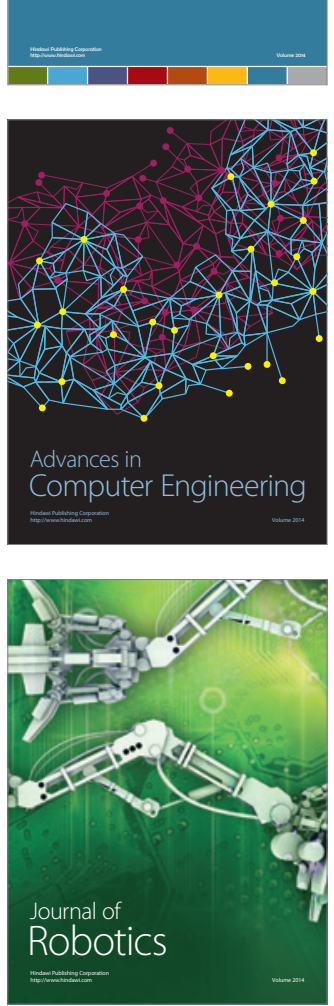
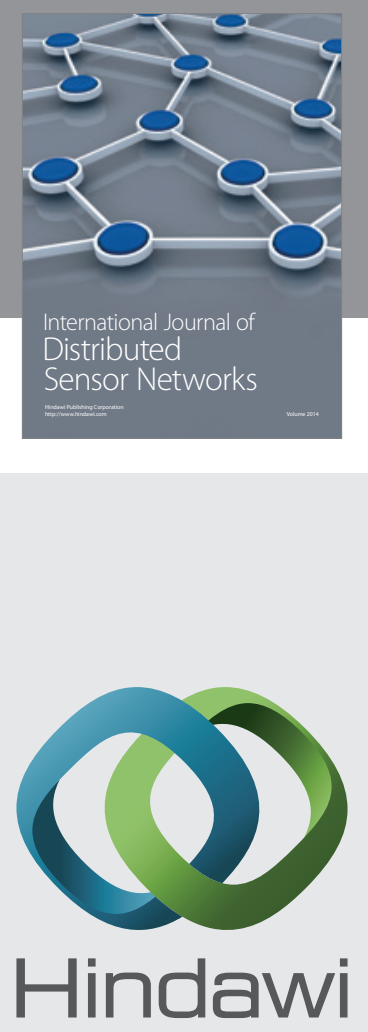

Submit your manuscripts at

http://www.hindawi.com
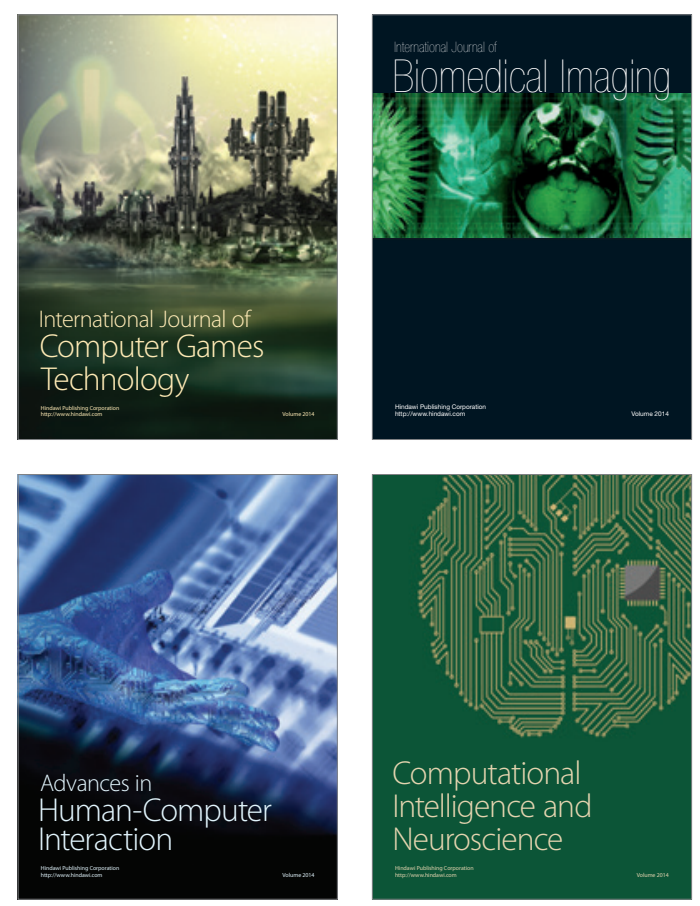
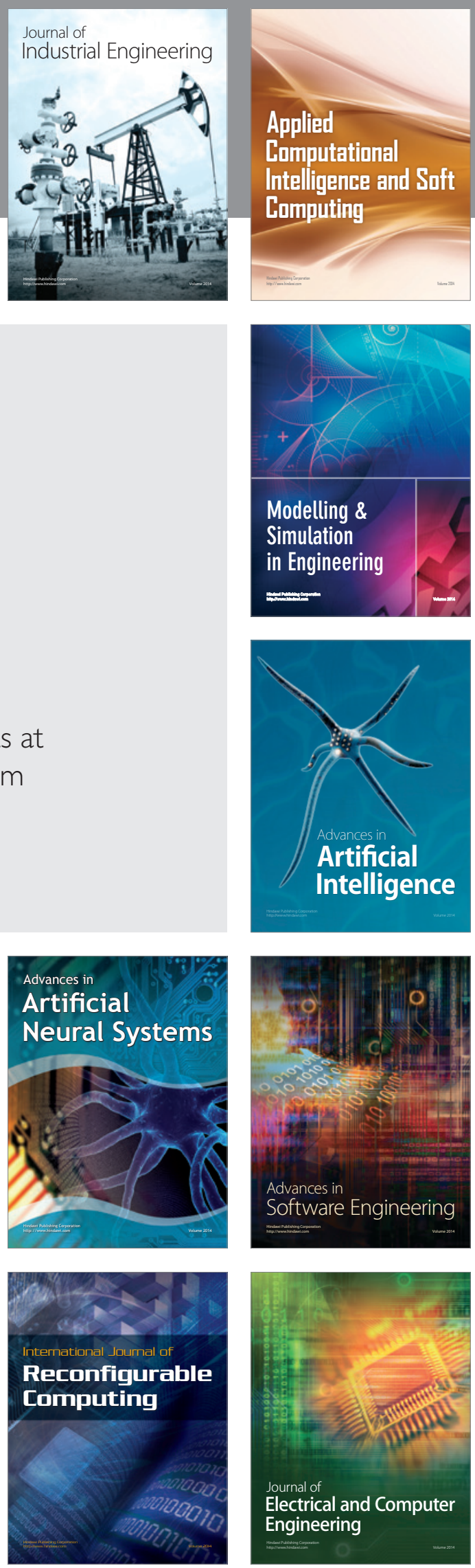